\title{
Tracing the coronal emission in Circinus with VLT/NACO
}

\author{
Olivier Marco ${ }^{1}$ and Almudena Prieto ${ }^{2}$ \\ ${ }^{1}$ ESO Paranal, Casilla 19001, Santiago, Chile email:omarco@eso.org \\ ${ }^{2}$ MPIA, Heidelberg, Germany email:prieto@mpia.de
}

\begin{abstract}
In this short letter we present our new work on the coronal emission in a sample of AGN. We used the adaptive optics system NACO on the VLT to obtain high angular resolution images $(\sim 0.15 ")$ of the coronal emission in the vicinity of the central engine.
\end{abstract}

\section{What and where is the coronal region in AGN?}

The coronal line region in AGN is a central nuclear region of gas where lines of very high ionization species are generated. Coronal lines are produced all across the electromagnetic spectrum. Some of the strongest ones accessible from the ground are the series of Fe lines

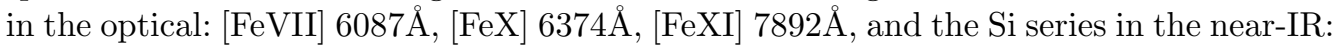
[SiVI] $1.96 \mu \mathrm{m},[\mathrm{SiVII}] 2.48 \mu \mathrm{m}$ and [SiIX] 3.93 $\mu \mathrm{m}$. Bright coronal emission lines [SiVI] and [Si VII] are common in Seyferts (Reunanen, Kotilanien \& Prieto 2003). Because of their high Ionization Potential (IP), these lines cannot be produced in starburst regions, but in extreme energetic environments such as an AGN. Indeed, the high ionization species that give rise to these lines can only survive very close to their ionization source, thus making them unique tracers of the AGN activity. Furthermore, they are also a clean diagnostic tool of the AGN energetic. For comparative purposes, both the Narrow- and Broad-line regions do have a substantial, sometimes dominant, contribution from circumnuclear star forming regions due to photoionization by $\mathrm{OB}$ stars or stellar winds and SN explosions. Coronal lines are free of that.

Due to the above, coronal lines can be used to directly pin point the AGN location and set limits on the AGN action radius. They also trace the regions of high nuclear gas density, as their critical density is about $10^{6} \mathrm{~cm}^{-3}$, and thus, their spatial morphology may reveal a connection with other AGN-related features, as jets, shocked-compressed regions. Finally, coronal lines can provide a direct measurement of the hardness and power of the AGN ionizing source. Furthermore, these lines are seen with similar strength and frequency in both Seyfert type (Prieto \& Viegas 2000), thus their study can provide a clean test of the Seyfert unification model.

Coronal line studies, however, have so far been hampered by the general weakness of these lines. They usually represent a few percent of the continuum emission, which makes them difficult to detect in seeing limited imaging or low resolution spectra. However, being these lines basically spatially unresolved, their study can largely benefit from high both spectral and spatial resolution.

The size of the broad line region is in the pc scale and therefore unresolved with current available classical instrumentation. The size of the coronal line region is within a few tens of pc (Contini, Prieto \& Viegas 1998) and this scale is now reachable at the diffraction limit of an $8 \mathrm{~m}$ telescope in the near-infrared. In this range, coronal lines can be seen rather strong in bright Seyfert galaxies, particularly if relatively high spatial resolution spectra are obtained. 


\section{Adaptive Optics to image CLR emission in AGN}

Many AO observations of AGN have been made so far, in particular to study the dust in the infrared (for example see Marco \& Alloin 1998) or to search for the torus (for example see Marco \& Alloin 2000 and Marco, Alloin \& Beuzit 1997). We have used the ESO/NACO adaptive optics system at the VLT to take images of several AGN, selected for their proximity and because coronal lines had previously been detected in spectroscopy. The spatial information is complementary to the spectral information, since for the first time the location of the CLR emission will be unambiguous. The pixel size is 0.027 " with a total field of view of $27 \times 27$ arcsec, allowing a precise registration of the various components.

In the case of Circinus, the physical scale traced by our images ranges between 1.5 to $3 \mathrm{pc}$, and therefore they can probe the presence and dimension of obscuring material around the AGN (for molecular material see for example Marco \& Brooks 2003 and Alloin et al. 2001, both for NGC 1068).

\section{3. [Si VII] emission as a tracer of the hidden nucleus}

We used Circinus as an archetypal case. Actually, in all the images of Circinus (both NACO \& HST) we observe some giant stars which can be identified unambiguously. We use them for absolute positioning of the images. Comparing the infrared wavelength J, $\mathrm{H}, \mathrm{K}$ and L images, with the HST WFPC2 broad-band image obtained with the filter F814W and the continuum subtracted [OIII] and $\mathrm{H} \alpha$ images, we are able to reconstruct a full set of high angular resolution images with a high precision astrometry $(\sim 1$ pixel of NACO: 0.027 arcsec). This allows us to build a true-color image of Circinus, combining HST H $\alpha$, NACO [Si VII] $2.48 \mu \mathrm{m}$ and NACO L band images. A spot due to coronal and hot dust emission clearly appears at the apex of the $\mathrm{H} \alpha$ ionization cone. This result is a clear demonstration that the [Si VII] $2.48 \mu \mathrm{m}$ can actually be used to pin point the active nucleus location. The source of excitation is photoionization by the central source UV \& X-ray. The extension of the coronal line region is on a parsec scale (10 pc diameter here). It is mixed with the hot dust and the NLR emission.

The results (including color images) presented here will be published in ApJ (Prieto et al. 2004).

\section{References}

Alloin, D., Galliano, E., Cuby, J. G., Marco, O., Rouan, D., Clénet, Y., Granato, G. L., \& Franceschini, A. 2001, A\&A, 369, L33

Contini, M., Prieto, M. A., \& Viegas, S. M. 1998, ApJ, 505, 621

Marco, O., \& Alloin, D. 2000, A\&A, 353, 465

Marco, O., \& Alloin, D. 1998, A\&A, 336, 823

Marco, O., Alloin, D., \& Beuzit, J. L. 1997, A\&A, 320, 399

Marco, O., \& Brooks, K. J. 2003, A\&A, 398, 101

Prieto, M. A., \& Viegas, S. M. 2000, ApJ, 532, 238

Prieto, M. A., et al. 2004, ApJ, in press (astro-ph/0406620)

Reunanen, J., Kotilainen, J. K., \& Prieto, M. A. 2003, MNRAS, 343, 192 